\title{
An Approximated Analytical Model for Pole-to-ground Faults in Symmetrical Monopole MMC-HVDC Systems
}

\author{
Vinícius A. Lacerda, Student Member, IEEE, Renato M. Monaro, Member, IEEE, David Campos-Gaona, Senior \\ Member, IEEE, Rafael Peña-Alzola, Senior Member, IEEE, Denis V. Coury
}

\begin{abstract}
Developing pole-to-ground (PG) fault models for Modular Multilevel Converters (MMC) is not straightforward due to the fault asymmetry and converter switching concerning blocking characteristics. Various studies have been carried out regarding transient simulation of PG faults. However, there is a lack of analytical models for the first stage of the fault. Therefore, this work proposes an approximated analytical model for PG faults in half-bridge MMCs. Closed-form expressions for the MMC contribution to the fault and the fault current are derived. We show that separating the solutions in different resonant frequencies represents the system dynamics and facilitates the interpretation of the phenomena. When compared to system calculated by Ordinary Differential Equations (ODEs), the proposed model provided a good approximation for a wide range of parameters. When compared to the full PSCAD solution, the analytical model was able to precisely calculate the peak fault current value, which confirmed its validity.
\end{abstract}

Keywords-Analytical model, MMC, Pole-to-ground fault, Short-circuit, VSC-HVDC.

\section{INTRODUCTION}

$\mathbf{M}$ ODULAR multilevel converters (MMCs) are viewed as an enabling technology for the transition from $\mathrm{AC}$ grids to multiterminal High-Voltage Direct Current (HVDC) systems, shaping the grid of the future concerning the use of renewables and allowing the interconnection of asynchronous systems [1].

However, challenges such as DC grid control and protection must be overcome before transitioning from point-to-point HVDC technology to multiterminal systems [2]. In order to design reliable and stable DC grids, control and protection systems must operate properly when the system is subjected to pole-to-ground (PG) or pole-to-pole (PP) faults. Thus, the system behaviour during faults must be well understood, which is crucial in designing control systems for unbalanced conditions, as in [3]-[6]. Although numerical models and simulations are more precise than approximated analytical models, a more in-depth understanding of the system behaviour is achieved when analytical models are used, which highlights its importance.

Developing fault models for MMC-HVDC systems is a complex task. As the MMC submodules can operate in

This work was funded in part by the São Paulo Research Foundation (FAPESP), [grant number 2015/21167-6].

V. A. Lacerda, R. M. Monaro and D. V. Coury are with the University of São Paulo, Brazil. (e-mail: vinicius.albernaz.freitas@usp.br; monaro@usp.br; coury@sc.usp.br). D. Campos-Gaona and R.Peña-Alzola are with the University of Strathclyde, Glasgow, Scotland. (e-mail: rafael.pena-alzola@strath.ac.uk; d.campos-gaona@strath.ac.uk) different states: inserted, bypassed or blocked, the fault model must be separated into different time periods where the equations defining the fault dynamics can be simplified [7]-[10]. It is well known that PP faults result in higher fault currents than PG faults and their equivalent circuit model is simpler than for PG faults because of the fault symmetry. In this context, various studies have been published regarding PP faults [7]-[9], [11]-[14]. However, PG faults are much more likely to occur than PP faults [15] and the topological differences between both types of faults prevent the PP fault models being used directly in PG faults. Thus, PG fault analytical models should also be studied.

Accordingly, [15] analyses the influence of wind power plant control methods in PG faults. The study was performed considering detailed models, but focusing on the stage after converter blocking. A similar study was performed by [16], where approximated analytical expressions were derived for the contribution of the $\mathrm{AC}$ grid to $\mathrm{PG}$ faults after the converters were blocked. The influence of MMC control on the fault currents is considered in [17]. However, the study focused on developing Ordinary Differential Equations (ODEs) that describe the multiterminal system fault dynamics, which are useful for numerical evaluation, but did not provide an analytical solution. In [18], the transient behaviour of a multiterminal HVDC system during PG faults was analysed using simulations but no analytical equations were given.

Precise models that consider the details of the system dynamics can be used [19], [20]. However, due to the system size and complexity, the analytical solutions can become complex or lose interpretability. Thus, this research provides approximated analytical solutions that can model the PG fault dynamics, preserving the frequency content and waveform of the variables during the first stage of the fault. We show that the system solution can be separated in different resonant frequencies, which represents the system dynamics and facilitates the interpretation of the phenomena. This paper focuses on symmetrical monopole configuration with half-bridge MMCs, because it is the most common MMC-HVDC configuration [21].

The main contribution of the paper is the development of a precise analytical pole-to-ground fault model, which can be used in HVDC studies, e.g. design of DC reactors and DC breakers, providing the participation of each system parameter in the system currents and voltages. The paper is organized as follows. Section II introduces characteristics of the half-bridge 
MMC subjected to PG faults and describes the proposed circuit model. Section III presents the approximated analytical solution. Section IV presents the assessment of the proposed model. Finally, conclusions are drawn in Section V. In order to provide seamless reproducibility, the equations and data in Section III are available in [22].

\section{THE MMC DURING A POLE-TO-GROUND FAULT}

A three-phase circuit of a half-bridge MMC subjected to $\mathrm{PG}$ faults is shown in Fig. 1. The three-phase MMC comprises six arms; three on the top and three on the bottom. Each arm is formed by an arm inductor $\left(L_{\text {arm }}\right)$ and $N$ series-connected submodules (SMs). The arm losses are represented by $R_{a r m}$. The DC side equivalent capacitance (submarine cable capacitance and DC side filter, if fitted) is represented by $C_{b}$. Fig. 1 also shows the $\mathrm{AC}$ grounding, represented by the star-point-reactor (SPR), though other AC grounding schemes may also be used, e.g. a zig-zag transformer [23], [24]. The SPR is used to provide a ground path in the converter's AC side and to rebalance any voltage unbalances due to control or monopole faults that could cause DC current flow in the transformers [23]. The SPR phase inductance $\left(L_{s p r}\right)$ is chosen to provide a low-impedance path for the DC current and a high-impedance path for the $\mathrm{AC}$ current [25]. The SPR resistor $\left(R_{s p r}\right)$ is used to mitigate any resonance between the SPR and the system [25].

Without loss of generality, all the analyses of this work are performed considering that the fault happens on the positive pole, which can be also applied to the negative pole, using their symmetry.

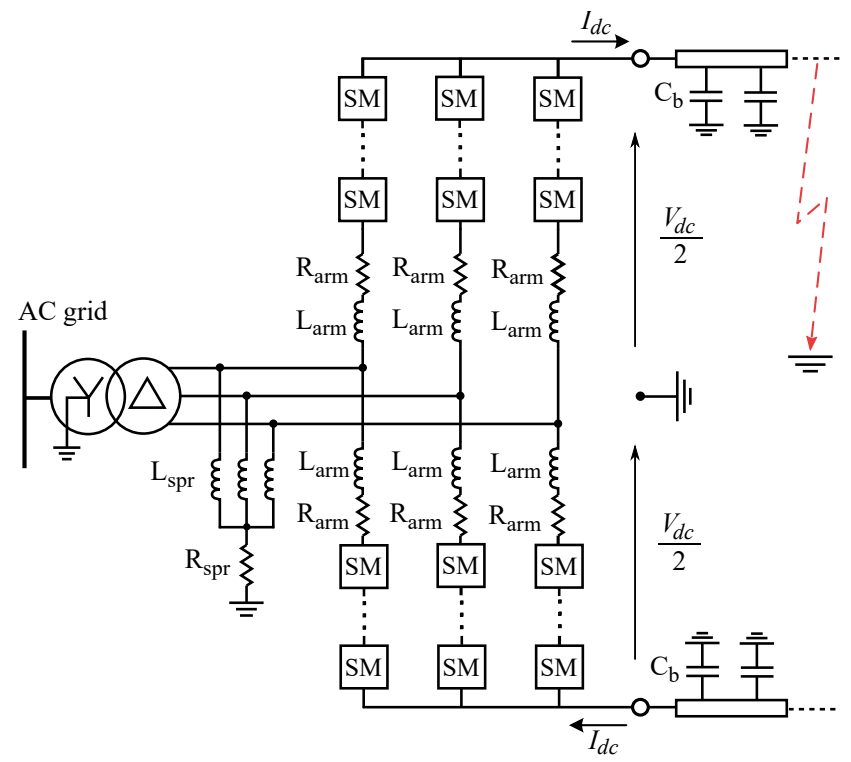

Fig. 1. MMC circuit diagram subjected to faults.

The MMC fault analysis is commonly divided into three stages [7], [8]. The first is the capacitive discharge stage, that is the period between the fault inception and the and the blocking of the converter. After the converter is blocked, the $\mathrm{AC}$ transient infeed stage starts, in which the energy stored in the arm inductors is released into the fault. After the inductors discharge the stored energy, the fault is fed by the AC grid in the AC steady-state infeed stage. This work analyses the first stage, as its understanding is crucial for control and protection systems.

A pole-to-ground (PG) fault may happen in overhead lines by direct or indirect contact with the ground, and, in cables by damage of the insulation. In overhead lines, the fault path can be established directly, from the conductor to the ground, or indirectly, through the tower. In cables, the fault path is established from the conductor to the sheath to the next grounding point [19], [26].

In order to define a proper equivalent circuit for the faulted system, the influence of each component in the fault current must be analysed. To simplify the circuit, components that do not affect the fault behaviour can be neglected without compromising the model precision. Although some consequences of PG faults on DC systems are well known, e.g., the voltage drop in the faulted pole and voltage rise in the non-faulted pole [24], [26], better understanding is needed regarding the variables and system configurations that can affect the fault behaviour. Considering this, the influence of the grounding and fault time instant on the fault behaviour is analysed next. Then, the equivalent circuit model of the faulted system is presented.

\section{A. Influence of grounding}

As the interface transformer is commonly connected in delta on the converter side, the fault loop is formed between the fault point and the DC side capacitance and the SPR (or other AC grounding scheme). If both grounding points are removed, the AC voltage neutral point will be shifted to the pole DC voltage value (Fig. 2) and the fault current will have no contribution. This behaviour, although desirable for the current, is prohibitive because of the large overvoltage imposed to the system [21].

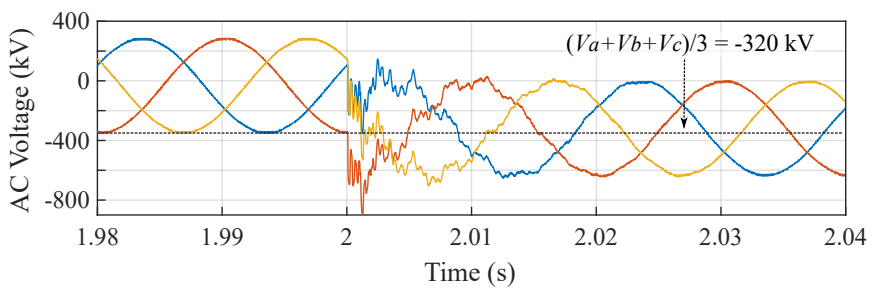

Fig. 2. MMC AC voltage during a PG fault, without a grounding point.

The SPR provides a fixed grounding reference on the AC side. However, $L_{s p r}$ is chosen to provide a high-impedance path for non-DC currents. As in the first milliseconds the fault currents are composed invariably of non-DC components (mix of oscillatory components and exponentially decaying DC components), the SPR presents high-impedance values to these currents. Thus, in the capacitive discharge state, the SPR current contribution to the fault can be neglected and the ground path formed by it can be considered an open-circuit.

The low-impedance path is provided by the DC side capacitance. When a fault happens, the energy stored in the line's/cable's capacitance or in another DC capacitor is discharged into the fault showing oscillatory behaviour. Therefore, the DC side capacitance must be taken into account during the first stage of the fault. 


\section{B. Influence of the fault time instant}

As the equivalent capacitance in each arm varies over time, preliminary analysis on the fault behaviour could presume that the dynamics would be influenced by the instant the fault happens. However, while this might be true for a single-phase MMC, it does not hold for a three-phase MMC. Let $n_{p}^{a}, n_{p}^{b}$ and $n_{p}^{c}$ be the number of inserted capacitors at instant $t$ on positive arms of phases $\mathrm{A}, \mathrm{B}$ and $\mathrm{C}$, respectively, and let $v_{u}$ and $v_{l}$ be the upper and lower arms voltages, respectively. Moreover, let $N$ be the total number of SMs in each arm. The number of inserted SMs is given by [27]:

$$
n_{p}^{x}=N \frac{v_{c}^{x}-v_{s}^{x}}{v_{p}^{\Sigma}}
$$

where $x=a, b, c$ indicates the three phases, $v_{c}=\left(v_{u}+v_{l}\right) / 2$ is the internal voltage, $v_{s}$ is the output voltage and $v_{p}^{\Sigma}$ is the sum of the voltages in each positive arm. Thus, assuming that the converter is generating balanced output voltages at frequency $\omega_{g}, v_{c}$ is balanced and equal to $V_{d c} / 2$ and $v_{p}^{\Sigma}$ is equal to $V_{d c}$, the three-phase output voltages are:

$$
\begin{aligned}
v_{s}^{a} & =\frac{1}{2} V_{d c} \cos \omega_{g} t \\
v_{s}^{b} & =\frac{1}{2} V_{d c} \cos \omega_{g} t-2 \pi / 3 \\
v_{s}^{c} & =\frac{1}{2} V_{d c} \cos \omega_{g} t+2 \pi / 3
\end{aligned}
$$

Taking the average number of inserted SMs $\left(n_{p}^{a v g}\right)$, it yields:

$$
n_{p}^{a v g}=\sum_{x=a, b, c} \frac{n_{p}^{x}}{3}=\frac{N}{3} \sum_{x=a, b, c} \frac{V_{d c} / 2-v_{s}^{x}}{V_{d c}}=N / 2
$$

Equation (3) shows that the average number of inserted SMs across the three phases of positive and negative arms is $N / 2$. Hence, assuming that all submodules are charged with the same voltage, the average voltage generated by the MMC on the DC side is $V_{d c} / 2$, regardless of the fault time instant. Thus, if the aforementioned conditions are true, no difference will be observed if two identical faults are simulated at different time instants. If the submodules are not balanced or alternative control methods or fault current suppression method such as [11], [28], [29] are used, the result of (3) might change.

In order to confirm this result, Fig. 3a shows $n_{p}^{a v g}$ during faults that were simulated with $1 \mathrm{~ms}$ of difference up to a range of $40 \mathrm{~ms}$ (in the system described in Section IV). It can be noticed that $n_{p}^{a v g}$ remains close to $N / 2$, according to (3). As a result, Fig. $3 \mathrm{~b}$ shows that the current waveform is almost the same, regardless of the fault instant. The waveforms were shifted in time to align them with respect to the fault instant.

\section{The equivalent circuit model for the arms}

When defining circuit models for PP faults, the converter is commonly modelled as three branches of equivalent capacitors in series with $L_{a r m}$ and $R_{a r m}$. The initial condition for the voltage of the equivalent capacitor is considered equal to $V_{d c} / 2$. This model assumes that the submodule capacitors do not charge during the fault and that they only discharge into the fault. Although this assumption is correct for PP faults, it may not be correct for PG faults.
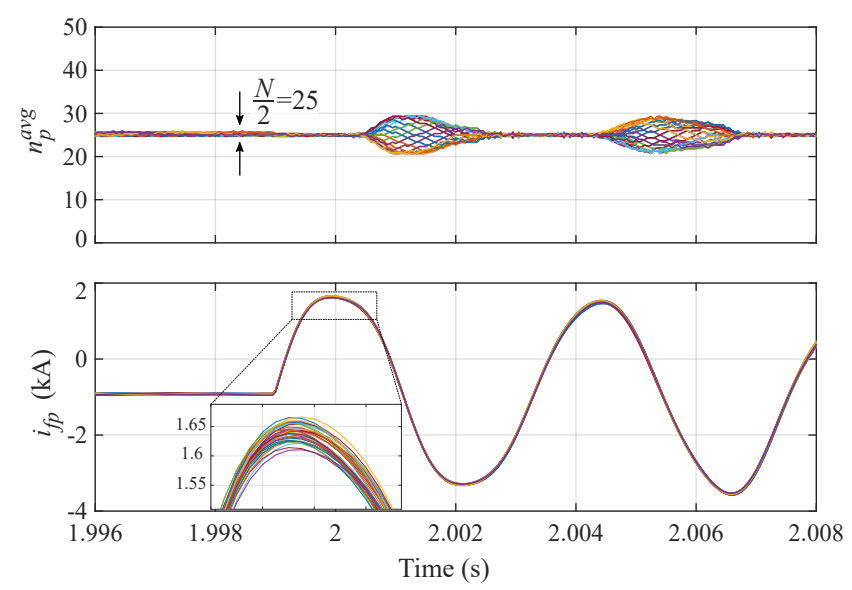

Fig. 3. Superposition of waveforms recorded at different fault instants. a) average number of inserted SMs on the positive arms and b) fault current.

Even considering that $n_{p}^{a v g}=N / 2$, one cannot define a unique equivalent capacitance for the positive or negative arms, regardless of time. This happens because of the non-linear relation defined by the series equivalent of capacitances. As the inverse of the series equivalent is equal to the sum of the inverses of each capacitance, the arms equivalent capacitances will change non-linearly over time, as well as their sum.

Another difference between converter behaviour during PG and PP faults is the fault path. In a PP fault, a short-circuit is formed between both arms and the fault. Thus, the converter stored energy is dissipated into the fault. On the other hand, in a PG fault, no short-circuit path is established between the arms and the fault, and the converter does not discharge into the fault. In this case, the converter energy is kept constant with slight variations. This difference between PP and PG faults with respect to the stored energy inside the converter can be observed when the sum of voltage in the SMs of positive $v_{p}^{\Sigma}$ and negative $v_{n}^{\Sigma}$ arms are analysed. As Fig. 4 shows, $v_{p}^{\Sigma}$ and $v_{n}^{\Sigma}$ hold their value while in the PP fault $v_{p}^{\Sigma}$ and $v_{n}^{\Sigma}$ drop following a capacitor discharge behaviour. In this example, the converter was intentionally not blocked to emphasize the difference in the discharge pattern due to PP and PG faults, but in a real case, the converter would be blocked before the submodules voltage collapses to zero.
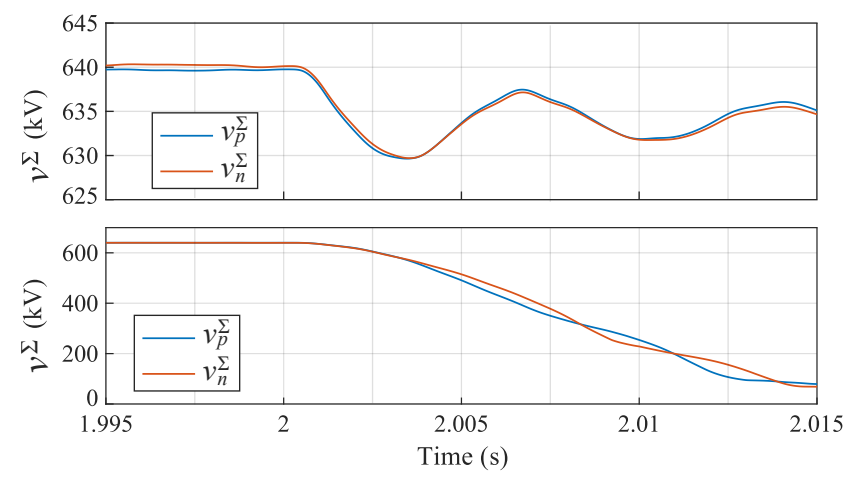

Fig. 4. Sum of SM voltages in positive and negative arms compared to the average converter voltage a) PG fault and b) PP fault.

As the converter keeps the voltage during a PG fault, the simplest circuit representation for each arm would be a DC 
voltage source equal to $V_{d c} / 2$ in series with $L_{a r m}$ and $R_{a r m}$. Though we acknowledge that the control will influence the arms voltages, this influence is less prominent in the first milliseconds of the fault [30].

\section{APPROXIMATED ANALYTICAL SOLUTION}

The circuit model resulting from the hypotheses discussed in Section II is shown in Fig. 5. Differently from PP faults, in which a short-circuit model can be established considering only one converter, in PG faults both converters connected to the faulted line must be considered. If only one converter is considered, there will be no return path for the currents at the sound pole, which will affect the precision of the model. Another approximation of the model in Fig. 5 is that the voltage at all arms on both converters is $V_{d c} / 2$. This consideration would lead to a zero pre-fault steady-state current $\left(I_{d c}=0\right)$ in both converters. However, $I_{d c}$ can be added later to the solution with no loss of precision. Although the DC side capacitance is distributed among the cables/lines and the terminal capacitances, in the approximated model of Fig. 5 it was considered concentrated in $C_{b}$.

In order to allow seamless reproducibility, the full equations and data associated with this model are available in [22].

The circuit elements of Fig. 5 are defined as follows: $R_{a}=R_{a r m} / 3, L_{a}=L_{a r m} / 3, C_{b}$ is the DC bus capacitor, $L_{d c}$ represents the conductor series inductance plus any DC inductor used to limit the fault current, $R_{d c}$ represents the losses in $L_{d c}$ and $R_{f}$ is the fault resistance. The subscripts 1 and ${ }_{2}$ refer to terminals 1 and 2, respectively.

Applying Kirchhoff's voltage and current laws to the circuit of Fig. 5 produces the following system of ODEs

$$
\begin{aligned}
& \frac{\mathrm{d} i_{c 1}}{\mathrm{~d} t}=\frac{V_{d c}-v_{b p 1}-v_{b n 1}}{L_{a 1}}-\frac{R_{a 1}}{L_{a 1}} i_{c 1} \\
& \frac{\mathrm{d} i_{b p 1}}{\mathrm{~d} t}=\frac{v_{b p 1}+v_{b n 1}-V_{d c}+R_{a 1} i_{c 1}}{L_{a 1}}+\frac{v_{b p 1}-R_{d c 1} i_{f p 1}-R_{f} i_{f t}}{L_{d c 1}} \\
& \frac{\mathrm{d} i_{b n 1}}{\mathrm{~d} t}=\frac{v_{b p 1}+v_{b n 1}-V_{d c}+R_{a 1} i_{c 1}}{L_{a 1}}+\frac{v_{b n 1}-v_{b n 2}-R_{d c} i_{f n 1}}{L_{d c}} \\
& \frac{\mathrm{d} i_{c 2}}{\mathrm{~d} t}= \frac{V_{d c}-v_{b p 2}-v_{b n 2}}{L_{a 2}}-\frac{R_{a 2}}{L_{a 2}} i_{c 2} \\
& \frac{\mathrm{d} i_{b p 2}}{\mathrm{~d} t}=\frac{v_{b p 2}+v_{b n 2}-V_{d c}+R_{a 2} i_{c 2}}{L_{a 2}}+\frac{v_{b p 2}-R_{d c 2} i_{f p 2}-R_{f} i_{f t}}{L_{d c 2}} \\
& \frac{\mathrm{d} i_{b n 2}}{\mathrm{~d} t}=\frac{v_{b p 2}+v_{b n 2}-V_{d c}+R_{a 2} i_{c 2}}{L_{a 2}}+\frac{v_{b n 2}-v_{b n 1}-R_{d c} i_{f n 2}}{L_{d c}} \\
& \frac{\mathrm{d} v_{b p 1}}{\mathrm{~d} t}=-\frac{1}{C_{b 1}} i_{b p 1} \\
& \frac{\mathrm{d} v_{b n 1}}{\mathrm{~d} t}=-\frac{1}{C_{b 1}} i_{b n 1} \\
& \frac{\mathrm{d} v_{b p 2}}{\mathrm{~d} t}=-\frac{1}{C_{b 2}} i_{b p 2} \\
& \frac{\mathrm{d} v_{b n 2}}{\mathrm{~d} t}=-\frac{1}{C_{b 2}} i_{b n 2}
\end{aligned}
$$

where, $L_{d c}=L_{d c 1}+L_{d c 2}, R_{d c}=R_{d c 1}+R_{d c 2}$ and $i_{f t}=$ $i_{f p 1}+i_{f p 2}$. As (4) is linear, the system has a solution. However, the size of the problem makes an exact closed-form solution unfeasible.

The system in (4) can be transformed to the frequency domain using the Laplace transform and then simplified to obtain an approximated analytical solution. In the Laplace transform, the initial condition for the voltages is $V_{d c} / 2$ and for the currents is 0 , following the considerations described at the beginning of this Section. For the sake of simplicity, only the development and solutions for $i_{c 1}, i_{b p 1}$ and $i_{b n 1}$ are presented. The currents $i_{c 2}, i_{b p 2}$ and $i_{b n 2}$ are obtained by symmetry and the voltages are obtained using $(4 \mathrm{~g})$ to $(4 \mathrm{j})$.

At this point, it is convenient to define frequencies and time constants to represent the expressions in the frequency domain in the most compact form. Let the system equivalent frequencies be:

$$
\left\{\begin{array} { l } 
{ \omega _ { a 1 } = \frac { 1 } { \sqrt { C _ { b 1 } L _ { a 1 } } } } \\
{ \omega _ { d c 1 } = \frac { 1 } { \sqrt { C _ { b 1 } L _ { d c } } } \quad \text { and } } \\
{ \omega _ { 1 d c 1 } = \frac { 1 } { \sqrt { C _ { b 1 } L _ { d c 1 } } } }
\end{array} \left\{\begin{array}{l}
\omega_{a 2}=\frac{1}{\sqrt{C_{b 2} L_{a 2}}} \\
\omega_{d c 2}=\frac{1}{\sqrt{C_{b 2} L_{d c}}} \\
\omega_{2 d c 2}=\frac{1}{\sqrt{C_{b 2} L_{d c 2}}}
\end{array}\right.\right.
$$

and let the system equivalent time constants be:

$$
\left\{\begin{array} { l } 
{ \tau _ { a 1 } = \frac { 2 L _ { a 1 } } { R _ { a 1 } } } \\
{ \tau _ { f 1 } = \frac { 2 L _ { d c 1 } } { R _ { f } } } \\
{ \tau _ { a 1 R } = \frac { 2 L _ { d c } } { R _ { a 1 } } } \\
{ \tau _ { a 1 L } = \frac { 2 L _ { a 1 } } { R _ { d c } } }
\end{array} \quad \left\{\begin{array} { l } 
{ \tau _ { a 2 } = \frac { 2 L _ { a 2 } } { R _ { a 2 } } } \\
{ \tau _ { f 2 } = \frac { 2 L _ { d c 2 } } { R _ { f } } } \\
{ \tau _ { a 2 R } = \frac { 2 L _ { d c } } { R _ { a 2 } } } \\
{ \tau _ { a 2 L } = \frac { 2 L _ { a 2 } } { R _ { d c } } }
\end{array} \text { and } \left\{\begin{array}{l}
\tau_{d c 1}=\frac{2 L_{d c 1}}{R_{d c 1}} \\
\tau_{d c 2}=\frac{2 L_{d c 2}}{R_{d c 2}} \\
\tau_{d c}=\frac{2 L_{d c}}{R_{d c}}
\end{array}\right.\right.\right.
$$

The Laplace transform of $i_{c 1}(t), i_{b p 1}(t)$ and $i_{b n 1}(t)$ are, respectively:

$$
\begin{aligned}
I_{c 1}(s) & =Q_{c 1}(s) / P(s) \\
I_{b p 1}(s) & =Q_{b p 1}(s) / P(s) \\
I_{b n 1}(s) & =Q_{b n 1}(s) / P(s)
\end{aligned}
$$

where $P(s)$ contains the system poles and $Q_{c 1}(s), Q_{b p 1}(s)$, $Q_{b n 1}(s)$ contain the system zeros. The complete expression of these variables can be found in [22].

Inverting (5) back to the time domain requires the roots of $P(s)$ to be known, so that $P(s)$ can be factored and the partial fraction expansion is applied. However, as it is not possible to find an algebraic solution with a polynomial of degree greater than four (see Abel-Ruffini Theorem), $P(s)$ has to be reduced to a lower degree polynomial. This can be achieved by neglecting the system resistances. If the system resistances are neglected, $P(s)$ reduces from:

$$
P(s)=p_{9} s^{9}+p_{8} s^{8}+p_{7} s^{7}+p_{6} s^{6}+p_{5} s^{5}+p_{4} s^{4}+p_{3} s^{3}+p_{2} s^{2}+p_{1} s+p_{0}
$$

to:

$$
P(s) \approx p_{8} s^{8}+p_{6} s^{6}+p_{4} s^{4}+p_{2} s^{2}+p_{0}
$$

and one can make the substitution $s^{2}=u$ and $P(u)$ becomes a polynomial of degree four, where $p_{8}=2$ and, 


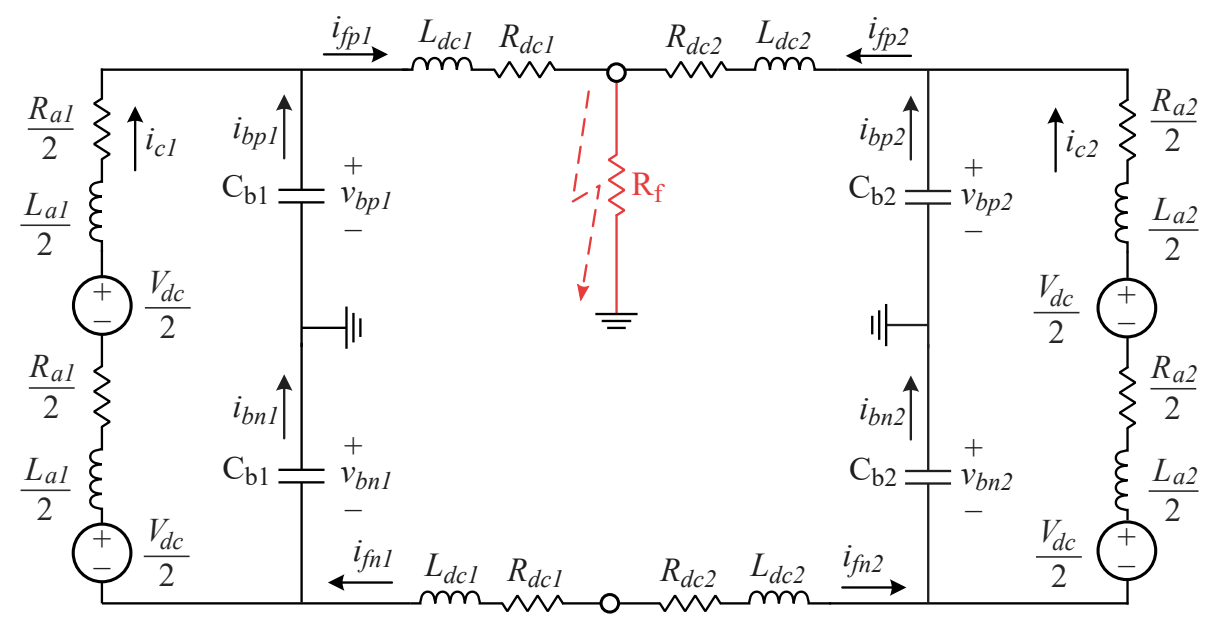

Fig. 5. System in PG fault at the capacitive discharge stage.

$$
\begin{aligned}
p_{6}= & 2 \omega_{1 d c 1}^{2}+2 \omega_{2 d c 2}^{2}+4 \omega_{a 1}^{2}+4 \omega_{a 2}^{2}+2 \omega_{d c 1}^{2}+2 \omega_{d c 2}^{2} \\
p_{4}= & 2\left(\omega_{1 d c 1}^{2}+\omega_{2 d c 2}^{2}\right)\left(\omega_{d c 2}^{2}+\omega_{d c 1}^{2}+2 \omega_{a 2}^{2}+2 \omega_{a 1}^{2}\right) \\
& +4\left(\omega_{a 1}^{2}+\omega_{a 2}^{2}\right)\left(\omega_{d c 2}^{2}+\omega_{d c 1}^{2}\right)+8 \omega_{a 1}^{2} \omega_{a 2}^{2}+2 \omega_{1 d c 1}^{2} \omega_{2 d c 2}^{2} \\
& -2 \omega_{a 1}^{2}\left(\omega_{1 d c 1}^{2}+\omega_{d c 1}^{2}\right)-2 \omega_{a 2}^{2}\left(\omega_{d c 2}^{2}+\omega_{2 d c 2}^{2}\right)
\end{aligned}
$$$$
p_{2}=2 \omega_{1 d c 1}^{2} \omega_{2 d c 2}^{2}\left(\omega_{a 1}^{2}+\omega_{a 2}^{2}+\omega_{d c 1}^{2}+\omega_{d c 2}^{2}\right)
$$$$
+4 \omega_{a 1}^{2} \omega_{a 2}^{2}\left(\omega_{1 d c 1}^{2}+\omega_{2 d c 2}^{2}+\omega_{d c 1}^{2}+\omega_{d c 2}^{2}\right)
$$$$
+2 \omega_{1 d c 1}^{2}\left(\omega_{a 1}^{2} \omega_{d c 2}^{2}+2 \omega_{a 2}^{2} \omega_{d c 1}^{2}+\omega_{a 2}^{2} \omega_{d c 2}^{2}\right)
$$$$
+2 \omega_{2 d c 2}^{2}\left(\omega_{a 1}^{2} \omega_{d c 1}^{2}+2 \omega_{a 1}^{2} \omega_{d c 2}^{2}+\omega_{a 2}^{2} \omega_{d c 1}^{2}\right)
$$

$$
\begin{aligned}
p_{0}=2 \omega_{1 d c 1}^{2} \omega_{2 d c 2}^{2} & \left(\omega_{a 1}^{2} \omega_{a 2}^{2}+\omega_{a 1}^{2} \omega_{d c 2}^{2}+\omega_{a 2}^{2} \omega_{d c 1}^{2}\right) \\
+ & 2 \omega_{a 1}^{2} \omega_{a 2}^{2}\left(\omega_{1 d c 1}^{2} \omega_{d c 2}^{2}+\omega_{2 d c 2}^{2} \omega_{d c 1}^{2}\right)
\end{aligned}
$$

$Q_{c 1}(s)$ reduces from a full 5-th order polynomial to:

where,

$$
Q_{c 1}(s)=\frac{V_{d c}}{C_{b 1} L_{a 1} L_{d c 1}}\left(s^{4}+q_{c 2} s^{2}+q_{c 0}\right)
$$

$$
\begin{aligned}
& q_{c 2}= \omega_{2 d c 2}^{2}+2 \omega_{a 2}^{2}+\omega_{d c 1}^{2}+\omega_{d c 2}^{2} \\
& q_{c 0}=\omega_{2 d c 2}^{2}\left(\omega_{a 2}^{2}+\omega_{d c 1}^{2}+\omega_{d c 2}^{2}-1 / \omega_{1 d c 1}^{2} \omega_{a 2}^{2} \omega_{d c 1}^{2}\right) \\
&+\omega_{a 2}^{2}\left(2 \omega_{d c 1}^{2}+\omega_{d c 2}^{2}\right)
\end{aligned}
$$

$Q_{b p 1}(s)$ reduces from a full 7-th order polynomial to:

$$
Q_{b p 1}(s)=\frac{V_{d c}}{L_{d c 1}}\left(s^{6}+q_{p 4} s^{4}+q_{p 2} s^{2}+q_{p 0}\right)
$$

where,

$$
\begin{aligned}
q_{p 4}= & \omega_{2 d c 2}^{2}+\omega_{a 1}^{2}+2 \omega_{a 2}^{2}+\omega_{d c 1}^{2}+\omega_{d c 2}^{2} \\
q_{p 2}= & \omega_{2 d c 2}^{2}\left(\omega_{a 1}^{2}+\omega_{a 2}^{2}+\omega_{d c 1}^{2}+\omega_{d c 2}^{2}\right) \\
& +\omega_{a 2}^{2}\left(2 \omega_{a 1}^{2}+2 \omega_{d c 1}^{2}+\omega_{d c 2}^{2}\right)+\omega_{a 1}^{2} \omega_{d c 2}^{2} \\
q_{p 0}= & \omega_{2 d c 2}^{2}\left(\omega_{a 1}^{2} \omega_{a 2}^{2}+\omega_{a 1}^{2} \omega_{d c 2}^{2}+\omega_{a 2}^{2} \omega_{d c 1}^{2}\right) \\
& +\omega_{a 1}^{2} \omega_{a 2}^{2}\left(\omega_{d c 2}^{2}+\omega_{2 d c 2}^{2} / \omega_{1 d c 1}^{2} \omega_{d c 1}^{2}\right)
\end{aligned}
$$

and $Q_{b n 1}(s)$ reduces from a full 5-th order polynomial to:

$$
Q_{b n 1}(s)=-\frac{V_{d c}}{C_{b 1} L_{a 1} L_{d c 1}}\left(s^{4}+q_{n 2} s^{2}+q_{n 0}\right)
$$

where,

$$
\begin{aligned}
q_{n 2}=\omega_{2 d c 2}^{2}+2 \omega_{a 2}^{2}+ & \omega_{d c 2}^{2}+\frac{\omega_{2 d c 2}^{2} \omega_{a 2}^{2}}{\omega_{1 d c 1}^{2} \omega_{a 1}^{2}} \omega_{d c 1}^{2} \\
q_{n 0}=\omega_{2 d c 2}^{2}\left(\omega_{a 2}^{2}+\omega_{d c 2}^{2}\right. & \left.+\frac{\omega_{a 2}^{2}}{\omega_{a 1}^{2}} \omega_{d c 1}^{2}\right) \\
& +\omega_{a 2}^{2}\left(\omega_{d c 2}^{2}+\frac{\omega_{2 d c 2}^{2}}{\omega_{1 d c 1}^{2}} \omega_{d c 1}^{2}\right)
\end{aligned}
$$

Considering this approximation and supposing the roots of $P(s)$ are $\omega_{1}^{2}, \omega_{2}^{2}, \omega_{3}^{2}, \omega_{4}^{2}$, such that:

$$
P(s)=2\left(s^{2}+\omega_{1}^{2}\right)\left(s^{2}+\omega_{2}^{2}\right)\left(s^{2}+\omega_{3}^{2}\right)\left(s^{2}+\omega_{4}^{2}\right)
$$

The inverse Laplace transform of (5) is:

$$
\begin{aligned}
& i_{c 1}(t)=\frac{V_{d c} / C_{b 1}}{L_{a 1} L_{d c 1}}\left(\frac{\sin \omega_{1} t}{k_{\omega 1}}+\frac{\sin \omega_{2} t}{k_{\omega 2}}+\frac{\sin \omega_{3} t}{k_{\omega 3}}+\frac{\sin \omega_{4} t}{k_{\omega 4}}\right) \\
& i_{b p 1}(t)=\frac{V_{d c}}{L_{d c 1}}\left(\frac{\sin \omega_{1} t}{k_{\omega 1}}+\frac{\sin \omega_{2} t}{k_{\omega 2}}+\frac{\sin \omega_{3} t}{k_{\omega 3}}+\frac{\sin \omega_{4} t}{k_{\omega 4}}\right) \\
& i_{b n 1}(t)=\frac{V_{d c} / C_{b 1}}{L_{a 1} L_{d c 1}}\left(\frac{\sin \omega_{1} t}{k_{\omega 1}}+\frac{\sin \omega_{2} t}{k_{\omega 2}}+\frac{\sin \omega_{3} t}{k_{\omega 3}}+\frac{\sin \omega_{4} t}{k_{\omega 4}}\right)
\end{aligned}
$$

where $k_{\omega 1}, k_{\omega 2}, k_{\omega 3}$ and $k_{\omega 4}$ are different in (19) as they are calculated using (20) and (21):

$$
\begin{aligned}
& k_{\omega 1}=\frac{2 \omega_{1}\left(\omega_{2}^{2}-\omega_{1}^{2}\right)\left(\omega_{3}^{2}-\omega_{1}^{2}\right)\left(\omega_{4}^{2}-\omega_{1}^{2}\right)}{-q_{6 x} \omega_{1}^{6}+q_{4 x} \omega_{1}^{4}-q_{2 x} \omega_{1}^{2}+q_{0 x}} \\
& k_{\omega 2}=\frac{2 \omega_{2}\left(\omega_{1}^{2}-\omega_{2}^{2}\right)\left(\omega_{3}^{2}-\omega_{2}^{2}\right)\left(\omega_{4}^{2}-\omega_{2}^{2}\right)}{-q_{6 x} \omega_{2}^{6}+q_{4 x} \omega_{2}^{4}-q_{2 x} \omega_{2}^{2}+q_{0 x}} \\
& k_{\omega 3}=\frac{2 \omega_{3}\left(\omega_{1}^{2}-\omega_{3}^{2}\right)\left(\omega_{2}^{2}-\omega_{3}^{2}\right)\left(\omega_{4}^{2}-\omega_{3}^{2}\right)}{-q_{6 x} \omega_{3}^{6}+q_{4 x} \omega_{3}^{4}-q_{2 x} \omega_{3}^{2}+q_{0 x}} \\
& k_{\omega 4}=\frac{2 \omega_{4}\left(\omega_{1}^{2}-\omega_{4}^{2}\right)\left(\omega_{2}^{2}-\omega_{4}^{2}\right)\left(\omega_{3}^{2}-\omega_{4}^{2}\right)}{-q_{6 x} \omega_{4}^{6}+q_{4 x} \omega_{4}^{4}-q_{2 x} \omega_{4}^{2}+q_{0 x}}
\end{aligned}
$$


TABLE I

$$
i_{c 1}\left\{\begin{array} { l } 
{ q _ { 6 x } = 0 } \\
{ q _ { 4 x } = 1 } \\
{ q _ { 2 x } = q _ { c 2 } } \\
{ q _ { 0 x } = q _ { c 0 } }
\end{array} i _ { b p 1 } \left\{\begin{array} { l } 
{ q _ { 6 x } = 1 } \\
{ q _ { 4 x } = q _ { p 4 } } \\
{ q _ { 2 x } = q _ { p 2 } } \\
{ q _ { 0 x } = q _ { p 0 } }
\end{array} i _ { b n 1 } \quad \left\{\begin{array}{l}
q_{6 x}=0 \\
q_{4 x}=1 \\
q_{2 x}=q_{n 2} \\
q_{0 x}=q_{n 0}
\end{array}\right.\right.\right.
$$

If the system losses are not neglected, the sinusoidal terms of (19) will be multiplied by exponential terms $e^{\frac{-t}{\tau_{1}}}, e^{\frac{-t}{\tau_{2}}}, e^{\frac{-t}{\tau_{3}}}$ and $e^{\frac{-t}{\tau_{4}}}$, they will have a phase summed in the argument, and $\omega_{1}, \omega_{2}, \omega_{3}$ and $\omega_{4}$ will be slightly shifted, where the time constants $\tau_{1}, \tau_{2}, \tau_{3}$ and $\tau_{4}$ are the real parts of the roots of the full $P(s)$.

As the reduced $P(s)$ has an algebraic solution, the undamped resonance frequencies $\omega_{1}, \omega_{2}, \omega_{3}$ and $\omega_{4}$ can be found exactly. Nevertheless, the size of the exact expressions would make them impractical. Therefore, it is useful to derive approximated expressions for the resonance frequencies. The first frequency $\left(\omega_{1}\right)$ can be derived by transforming the circuit to the frequency domain, calculating the Thévenin equivalent circuit connected to both $C_{b 1}$ and finding the frequency that results in equivalent impedance to be zero. Similarly, $\omega_{2}$ is obtained using the same procedure with $C_{b 2}$. Next, $\omega_{3}$ and $\omega_{4}$ are obtained using Vieta's formulas. Using this approach, the undamped resonance frequencies are:

$$
\begin{aligned}
\omega_{1} & =\sqrt{\omega_{a 2}^{2}+\frac{\omega_{d c 2}^{2}}{2}+\frac{\omega_{2 d c 2}^{2}}{2}+\sqrt{\left(\frac{L_{d c 1} \omega_{d c 2}^{2}}{2 L_{d c 2}}\right)^{2}+\omega_{a 2}^{4}}} \\
\omega_{2} & =\sqrt{\omega_{a 1}^{2}+\frac{\omega_{d c 1}^{2}}{2}+\frac{\omega_{2 d c 1}^{2}}{2}+\sqrt{\left(\frac{L_{d c 2} \omega_{d c 1}^{2}}{2 L_{d c 1}}\right)^{2}+\omega_{a 1}^{4}}} \\
\omega_{3} & =\sqrt{\frac{1}{2}\left(-\omega_{1}^{2}-\omega_{2}^{2}+a_{1}+\sqrt{\left(\omega_{1}^{2}+\omega_{2}^{2}-a_{1}\right)^{2}-a_{2}-a_{3}}\right)} \\
\omega_{4} & =\sqrt{\frac{1}{2}\left(-\omega_{1}^{2}-\omega_{2}^{2}+a_{1}-\sqrt{\left(\omega_{1}^{2}+\omega_{2}^{2}-a_{1}\right)^{2}-a_{2}-a_{3}}\right)}
\end{aligned}
$$

where,

$$
\begin{aligned}
& a_{1}=2\left(\omega_{d c 1}^{2}+\omega_{d c 2}^{2}+\omega_{a 1}^{2}+\omega_{a 2}^{2}\right)+\frac{L_{d c 2} \omega_{d c 1}^{2}}{L_{d c 1}}+\frac{L_{d c 1} \omega_{d c 2}^{2}}{L_{d c 2}} \\
& a_{2}=4 \frac{2 \omega_{a 1}^{2} \omega_{a 2}^{2}\left(\omega_{d c 1}^{2} \omega_{2 d c 2}^{2}+\omega_{d c 2}^{2} \omega_{1 d c 1}^{2}\right)}{\omega_{1}^{2} \omega_{2}^{2}} \\
& a_{3}=4 \frac{\omega_{1 d c 1}^{2} \omega_{2 d c 2}^{2}\left(\omega_{a 1}^{2} \omega_{d c 2}^{2}+\omega_{a 2}^{2} \omega_{d c 1}^{2}\right)}{\omega_{1}^{2} \omega_{2}^{2}}
\end{aligned}
$$

Similarly, approximated expressions for the time constants can be derived, approximating the equivalent circuit connected to each capacitor:

$$
\begin{aligned}
& \tau_{1}=1 / 2\left(\tau_{a 1 L}^{-1}+\tau_{d c}^{-1}\right)^{-1}+1 / 2\left(\tau_{a 1}^{-1}+\tau_{a 1 R}^{-1}\right)^{-1} \\
& \tau_{2}=1 / 2\left(\tau_{a 2 L}^{-1}+\tau_{d c}^{-1}\right)^{-1}+1 / 2\left(\tau_{a 2}^{-1}+\tau_{a 2 R}^{-1}\right)^{-1} \\
& \tau_{3}=2\left(\tau_{d c 1}^{-1}+\tau_{d c 2}^{-1}\right)^{-1} \\
& \tau_{4}=\left(\tau_{f 1}^{-1}+\tau_{f 2}^{-1}+\tau_{d c}^{-1}\right)^{-1}
\end{aligned}
$$

TEST SYSTEM PARAMETERS.

\begin{tabular}{l|l|l|l}
\hline$V_{d c}=640 \mathrm{kV}$ & $L_{a 1}=42.4 \mathrm{mH}$ & $L_{a 2}=56.5 \mathrm{mH}$ & $L_{d c 1}=115 \mathrm{mH}$ \\
$I_{d c}=0.5 \mathrm{kA}$ & $R_{a 1}=0.45 \Omega$ & $R_{a 2}=0.59 \Omega$ & $R_{d c 1}=1 \Omega$ \\
$R_{f}=1 \Omega$ & $C_{b 1}=4 \mu \mathrm{F}$ & $C_{b 2}=3 \mu \mathrm{F}$ & $L_{d c 2}=180 \mathrm{mH}$ \\
& & & $R_{d c 2}=2 \Omega$ \\
\hline
\end{tabular}

Finally, the pre-fault DC current can be considered by adding $I_{d c}$ to $i_{c 1}(t)$ and $-I_{d c}$ to $i_{c 2}(t)$.

Equation (19) reveals that $i_{c 1}(t), i_{b p 1}(t)$ and $i_{b n 1}(t)$ are a sum of four oscillations, and that the contribution of each oscillation to the final response is governed by system parameters, summarized in (20). This can be extended to $i_{c 2}(t), i_{b p 2}(t), i_{b n 2}(t)$ and to the voltages $v_{b p 1}(t), v_{b n 1}(t)$, $v_{b p 2}(t)$ and $v_{b n 2}(t)$. This is a benefit of the analytical solution, to demonstrate the behaviour of each system variable and to express in closed-form expressions the frequency of each oscillation. The system behaviour subjected to faults can then be used, for example, in protection and control systems, suppressing unwanted oscillations caused by a fault.

\section{AsSESSMENT OF THE PROPOSED MODEL}

In order to assess the precision of the proposed model, the expressions (19) to (27) were compared in time domain with the numerical solution of the system of ODEs in (4). The ODEs solver was the explicit Runge-Kutta $(4,5)$ formula pair with variable step and $10^{-6}$ absolute error tolerance. The system parameters are summarized in Table I. The results for $i_{c 1}(t), i_{b p 1}(t)$ and $i_{b n 1}(t)$ are shown in Fig. 6, which confirms that the proposed model is precise, even with slight deviations in the end due to the approximated exponential decays.
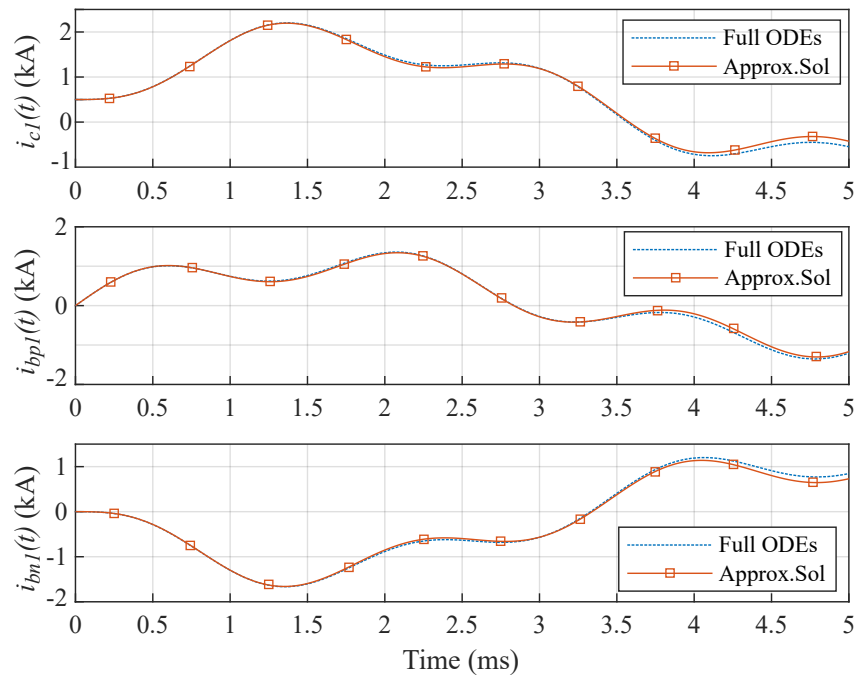

Fig. 6. Comparison in the time domain between the full numerical solution and the approximated solution. a) $i_{c 1}(t)$; b) $i_{b p 1}(t)$ and c) $i_{b n 1}(t)$.

To assess the validity of the proposed model not only for the parameters of Table I but also for a range of values, a parameter-sensitivity test was performed. The system's basic parameters $C_{b 1}, C_{b 2}, L_{a 1}, L_{a 2}, L_{d c 1}, L_{d c 2}$ and $R_{f}$ were varied in a range from $1 / 5$ to 5 times the values shown in Table I and the analytical solution and ODE numerical solution of $i_{c 1}, i_{b p 1}$ 

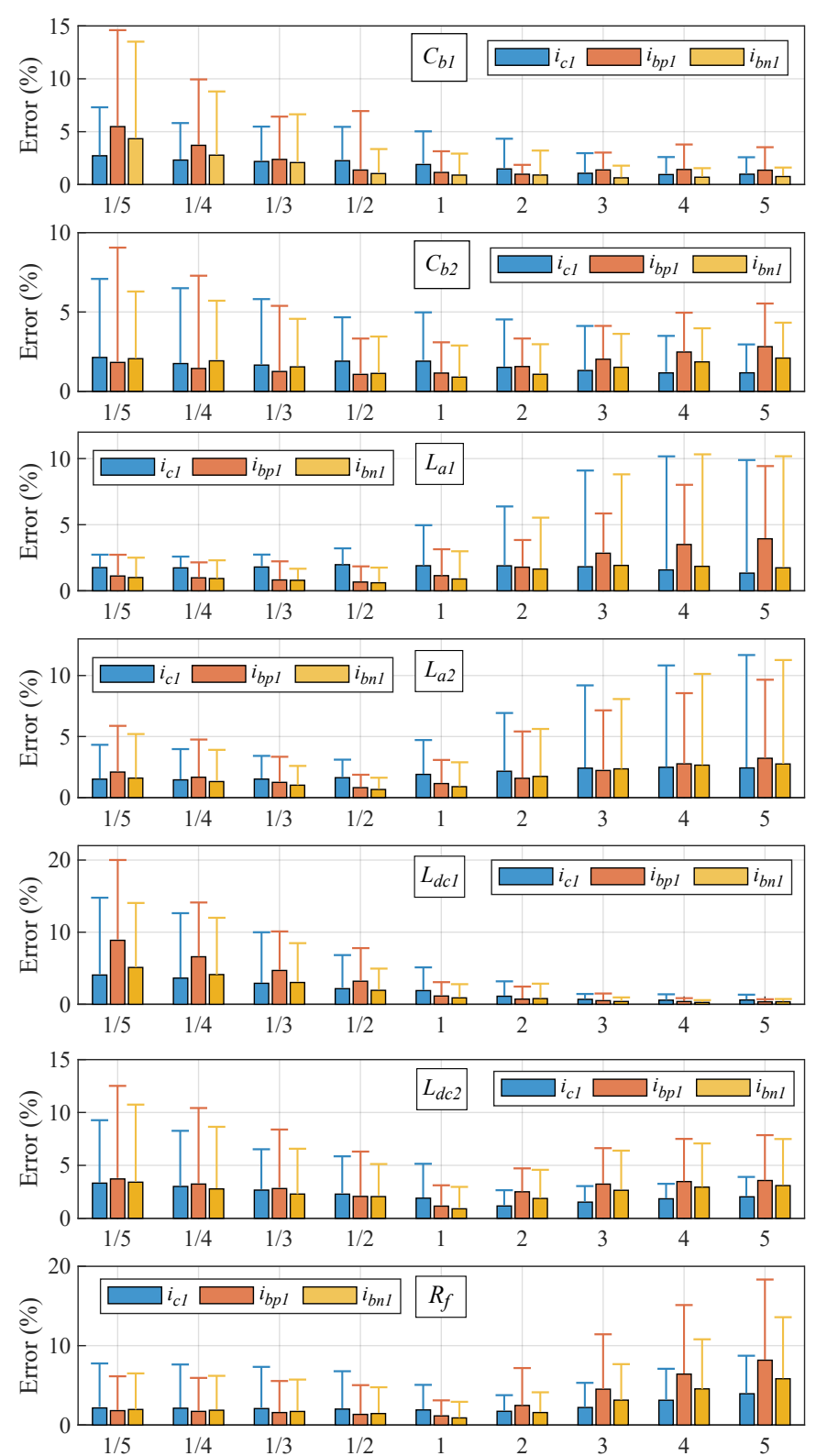

Fig. 7. Average error between the numerical ODEs solution and the approximated analytical solution of $i_{c 1}, i_{b p 1}$ and $i_{b n 1}$ for a range of parameters.

ad $i_{b n 1}$ were calculated and compared. The base $R_{f}$ was raised to $5 \Omega$ to increase the range of analysis. Then, the average error during the first $5 \mathrm{~ms}$ was taken and the relative error was calculated dividing the average error by the peak value of each variable, as shown in (28), where $e_{a v g}$ is the average error, $x_{a n}$ is the analytical solution, $x_{\text {ode }}$ is the numerical ODE solution and $N$ is the total number of samples. The results are shown in Fig. 7, where the bars represent the average error and the stems represent the maximum error.

$$
e_{\text {avg }}=\frac{1}{N} \sum_{k=1}^{N} \frac{\left|x_{\text {an }}(k)-x_{\text {ode }}(k)\right|}{\max \left(x_{\text {ode }}\right)}
$$

In Fig. 7, it can be observed that the approximated solution was valid not only for a specific set of parameters but also for a wide range of values. From $1 / 5$ to 5 , there is a difference of 25 times the parameter values, which highlights how the analyzed range was wide. In most cases, the average errors were less than $3 \%$ and the maximum average error was $5.1 \%$ for $C_{b 1}=0.8 \mu \mathrm{F}$. Variations in $C_{b 1}$ and $L_{d c 1}$ influenced the response more than the other parameters because $C_{b 1}$ and $L_{d c 1}$ are directly related to the resonant frequencies, which greatly influence the solution. The higher maximum errors were due to the approximated exponential decays, which increased the deviation near the end of the $5 \mathrm{~ms}$. Therefore, the results confirmed that the proposed equations can be used to calculate PG fault currents in MMC-HVDC systems that respect the solution hypotheses.

However, the ODEs do not represent other MMC-HVDC system characteristics, such as travelling waves, skin effect, lines' distributed parameters and converter control dynamics. Thus, to analyse if the validity of the proposed solution can also be extended to these scenarios, the approximated solution was compared to the fault waveforms of a two-terminal MMC-HVDC system, modelled in PSCAD. The system was adapted from [31]. The DC link was modelled using the frequency-dependent model. The line configuration was based on [32]. The converters were not blocked during the fault. The fault was simulated at $25 \mathrm{~km}$ from MMC 1 , with $R_{f}=5 \Omega$. The pre-fault values were $I_{d c}=0.5 \mathrm{kA}$ and $V_{d c}=640 \mathrm{kV}$. The DC inductors were equal to $40 \mathrm{mH}$ and $30 \mathrm{mH}$ for the MMC 1 and MMC 2, respectively.

When transforming the line frequency-dependent distributed parameters to fixed-frequency lumped parameters, some challenges arise. Figure 8 shows the variation of the line resistance and inductance with frequency.
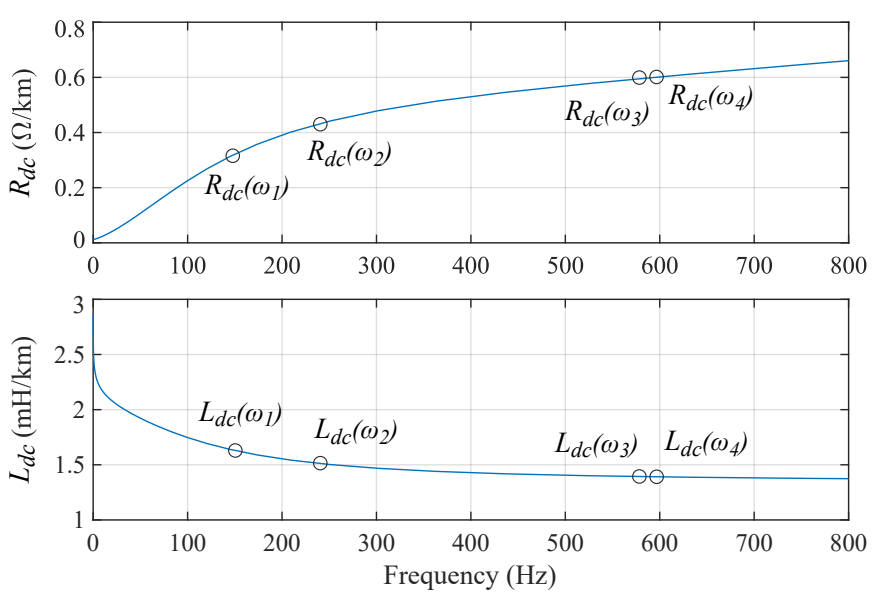

Fig. 8. Variation of line parameters with frequency. a) resistance; b) inductance.

As can be observed in Fig. 8, the lumped parameters taken from DC are different from the ones taken at each resonance frequency. If DC is chosen, the parameters will represent precisely the DC power flow but will not be precise in the transients, which have frequencies $\omega_{1}, \omega_{2}, \omega_{3}$ and $\omega_{4}$. If any of the resonant frequencies is chosen, the parameters will be precise for that frequency but will present errors for different ones. A simple approach to this problem is to consider specific $R_{d c 1}, L_{d c 1}, R_{d c 2}$ and $L_{d c 2}$ at each resonant frequency, calculate the oscillatory components separately using the specific parameters and then sum the response. Using 
this approach, the fault current $i_{f p 1}$ is shown in Fig. 9 in comparison to the PSCAD simulation.

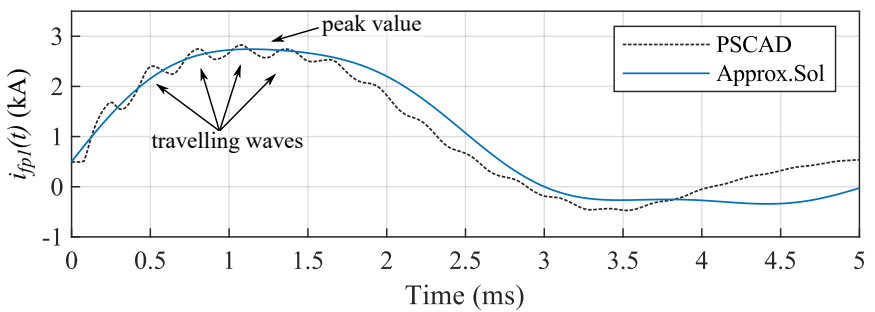

Fig. 9. Comparison in time domain between the PSCAD simulation and the approximated solution of $i_{f p 1}(t)$.

As can be observed in Fig. 9, the proposed approximated solution, although neglecting the travelling wave effect, was able to represent the first milliseconds of the fault, including the fault current peak value, an important parameter for protection system design. The accuracy of this value explains the previous assumptions, in which the resistances were neglected for the calculations. This in turn is a consequence of the PG fault, which does not result in great energy dissipation. The dominant frequency of $i_{f p 1}$ in the PSCAD simulation was calculated as $937.3 \mathrm{rad} / \mathrm{s}$, a value very close to $\omega_{4}=952.2 \mathrm{rad} / \mathrm{s}$ obtained with the analytical model.

\section{Conclusions}

Although transient simulations of PG faults in MMC-HVDC systems can be found in the literature, there is a lack of analytical models that describe the system dynamics subjected to faults. Therefore, this paper proposed an approximated analytical model for MMC-HVDC systems that represents the system dynamics during the converter capacitive discharge stage of PG faults. The analytical model revealed that the system dynamics can be described by a sum of four oscillatory components with defined resonant frequencies and exponential decays. The expressions for each resonant frequency and time constant were derived. Although numerical models and simulations are more accurate than approximated analytical models, the description of the system behaviour is an advantage of the latter. The described behaviour enhances the understanding about the system's control, protection and stability. Considering this, the importance of this study must be highlighted.

As obtaining an exact solution was not feasible due to the system size and complexity, approximations were made using hypotheses about the converters' control, grounding and steady-state values, as well as about the transmission line transient characteristics. When comparing to the system simulated by ODEs, the proposed model provided an accurate approximation for a vast range of system parameters. When comparing to the full PSCAD solution, the analytical model was able to precisely calculate the dominant resonant frequency and the peak fault current value, important parameters for the design of control and protection systems.

The presented contributions extend those found in the literature, providing a more in-depth understanding of the PG fault transient behaviour of MMC-HVDC systems with respect to the converter's voltages and currents.

\section{REFERENCES}

[1] D. V. Hertem, O. Gomis-Bellmunt, and J. Liang, Eds., HVDC Grids For Offshore and Supergrid of the Future. John Wiley \& Sons, Inc., feb 2016.

[2] D. Jovcic and K. Ahmed, High voltage direct current transmission: converters, systems and DC grids. John Wiley \& Sons, 2015.

[3] S. Li, X. Wang, Z. Yao, T. Li, and Z. Peng, "Circulating current suppressing strategy for MMC-HVDC based on nonideal proportional resonant controllers under unbalanced grid conditions," IEEE Trans. on Power Electronics, vol. 30, no. 1, pp. 387-397, jan 2015.

[4] G. Bergna-Diaz, J. A. Suul, E. Berne, J.-C. Vannier, and M. Molinas, "Optimal shaping of the MMC circulating currents for preventing AC-side power oscillations from propagating into HVdc grids," IEEE Journal of Emerging and Selected Topics in Power Electronics, vol. 7, no. 2, pp. 1015-1030, jun 2019.

[5] S. Cui, H.-J. Lee, J.-J. Jung, Y. Lee, and S.-K. Sul, "A comprehensive AC-side single-line-to-ground fault ride through strategy of an MMC-based HVDC system," IEEE Journal of Emerging and Selected Topics in Power Electronics, vol. 6, no. 3, pp. 1021-1031, sep 2018.

[6] J. Li, G. Konstantinou, H. R. Wickramasinghe, and J. Pou, "Operation and control methods of modular multilevel converters in unbalanced AC grids: A review," IEEE Journal of Emerging and Selected Topics in Power Electronics, vol. 7, no. 2, pp. 1258-1271, jun 2019.

[7] B. Li, J. He, J. Tian, Y. Feng, and Y. Dong, "DC fault analysis for modular multilevel converter-based system," Journal of Modern Power Systems and Clean Energy, vol. 5, no. 2, pp. 275-282, jan 2016.

[8] W. Leterme, J. Beerten, and D. V. Hertem, "Equivalent circuit for half-bridge MMC dc fault current contribution," in IEEE International Energy Conference (ENERGYCON), Leuven, Belgium, 2016.

[9] K. Satpathi, Y. M. Yeap, A. Ukil, and N. Geddada, "Short-time fourier transform based transient analysis of VSC interfaced point-to-point DC system," IEEE Transactions on Industrial Electronics, vol. 65, no. 5, pp. 4080-4091, may 2018.

[10] N. A. Belda, C. A. Plet, and R. P. P. Smeets, "Analysis of faults in multiterminal HVDC grid for definition of test requirements of HVDC circuit breakers," IEEE Transactions on Power Delivery, vol. 33, no. 1, pp. 403-411, feb 2018.

[11] R. Li, L. Xu, D. Holliday, F. Page, S. J. Finney, and B. W. Williams, "Continuous operation of radial multiterminal HVDC systems under DC fault," IEEE Transactions on Power Delivery, vol. 31, no. 1, pp. 351-361, feb 2016.

[12] C. Li, C. Zhao, J. Xu, Y. Ji, F. Zhang, and T. An, "A pole-to-pole short-circuit fault current calculation method for DC grids," IEEE Trans. on Power Systems, vol. 32, no. 6, pp. 4943-4953, nov 2017.

[13] B. Jiang and Y. Gong, "Arm overcurrent analysis and calculation of MMC-HVDC system with DC-link pole-to-pole fault," Electric Power Components and Systems, vol. 46, no. 2, pp. 177-186, jan 2018.

[14] X. Yang, Y. Xue, P. Wen, and Z. Li, "Comprehensive understanding of DC pole-to-pole fault and its protection for modular multilevel converters," High Voltage, vol. 3, no. 4, pp. 246-254, dec 2018.

[15] R. Vidal-Albalate, H. Beltran, A. Rolan, E. Belenguer, R. Pena, and R. Blasco-Gimenez, "Analysis of the performance of MMC under fault conditions in HVDC-based offshore wind farms," IEEE Transactions on Power Delivery, vol. 31, no. 2, pp. 839-847, apr 2016.

[16] M. K. Bucher and C. M. Franck, "Analytic approximation of fault current contribution from AC networks to MTDC networks during pole-to-ground faults," IEEE Transactions on Power Delivery, vol. 31, no. 1, pp. 20-27, feb 2016.

[17] M. Langwasser, G. D. Carne, M. Liserre, and M. Biskoping, "Fault current estimation in multi-terminal $\mathrm{HVdc}$ grids considering $\mathrm{MMC}$ control," IEEE Transactions on Power Systems, vol. 34, no. 3, pp. 2179-2189, may 2019.

[18] M. K. Bucher and C. M. Franck, "Analysis of transient fault currents in multi-terminal hvdc networks during pole-to-ground faults," in International Conference on Power Systems Transients (IPST), 2013.

[19] — "Analytic approximation of fault current contributions from capacitive components in HVDC cable networks," IEEE Transactions on Power Delivery, vol. 30, no. 1, pp. 74-81, feb 2015.

[20] M. Wang, J. Beerten, and D. V. Hertem, "Frequency domain based DC fault analysis for bipolar HVDC grids," Journal of Modern Power Systems and Clean Energy, vol. 5, no. 4, pp. 548-559, jul 2017.

[21] CIGRE Working Group B4-52, Technical Brochure 533: HVDC Grid Feasibility Study. CIGRE, 2013.

[22] V. A. Lacerda, R. M. Monaro, D. Campos-Gaona, R. Pena-Alzola, and D. V. Coury, "Dataset of the paper: An approximated analytical model 
for pole-to-ground faults in symmetrical monopole mmc-hvdc systems," https://github.com/valacerda/pgfaultmodel, 2020, GitHub repository.

[23] H. Wang, G. Tang, Z. He, and J. Yang, "Efficient grounding for modular multilevel HVDC converters (MMC) on the AC side," IEEE Trans. on Power Delivery, vol. 29, no. 3, pp. 1262-1272, jun 2014.

[24] M. Wang, W. Leterme, G. Chaffey, J. Beerten, and D. V. Hertem, "Pole rebalancing methods for pole-to-ground faults in symmetrical monopolar HVDC grids," IEEE Transactions on Power Delivery, vol. 34, no. 1, pp. 188-197, feb 2019.

[25] C. Armschat, M. Dommaschk, V. Hussennether, and T. Westerweller, "Star-point reactor," International Patent WO 2011/006796, 20 Jan 2011.

[26] F. B. Ajaei and R. Iravani, "Cable surge arrester operation due to transient overvoltages under DC-side faults in the MMC-HVDC link," IEEE Trans. on Power Delivery, vol. 31, no. 3, pp. 1213-1222, jun 2016.

[27] K. Sharifabadi, L. Harnefors, H.-P. Nee, S. Norrga, and R. Teodorescu, Design, Control, and Application of Modular Multilevel Converters for HVDC Transmission Systems. John Wiley \& Sons, 2016.

[28] X. Li, B. Zhao, Y. Wei, X. Xie, Y. Hu, and D. Shu, "DC fault current limiting effect of MMC submodule capacitors," International Journal of Electrical Power \& Energy Systems, vol. 115, p. 105444, feb 2020.

[29] V. A. Lacerda, R. M. Monaro, R. Peña-Alzola, D. Campos-Gaona, D. V. Coury, and O. Anaya-Lara, "Control-based fault current limiter for modular multilevel voltage-source converters," International Journal of Electrical Power \& Energy Systems, vol. 118, p. 105750, jun 2020.

[30] V. A. Lacerda, D. V. Coury, N. Y. Suzuki, and R. M. Monaro, "The impact of modular multilevel converter control on de short-circuit currents of hvdc systems," in Proceedings of the 20th Power Systems Computation Conference (PSCC), jun 2018.

[31] W. Leterme, N. Ahmed, D. V. Hertem, J. Beerten, S. Norrga, and L. Ängquist, "A new HVDC grid test system for HVDC grid dynamics and protection studies in EMT-type software," in 11th IET International Conference on AC and DC Power Transmission, Birmingham, UK, 2015.

[32] CIGRE Working Group B4-57, Technical Brochure 604: Guide for the Development of Models for HVDC Converters in a HVDC Grid. CIGRE, 2013. 